\title{
Canine mast cell tumors: diagnosis, treatment, and prognosis
}

This article was published in the following Dove Press journal:

Veterinary Medicine: Research and Reports

12 August 2014

Number of times this article has been viewed

\section{Laura D Garrett \\ Department of Veterinary Clinical Medicine, University of Illinois College of Veterinary Medicine, Urbana, IL, USA}

Correspondence: Laura D Garrett Department of Veterinary Clinical Medicine, University of Illinois College of Veterinary Medicine, 1008 W Hazelwood Dr, Urbana, IL 61802, USA

Tel +I 2172440194

Fax +I 2172449554

Email garrett I@illinois.edu

\begin{abstract}
Mast cell tumors (MCTs) are the most common malignant skin cancer in dogs, and significant variability exists in their biological behavior. Most MCTs are cured with appropriate local therapy, but a subset shows malignant behavior with the potential to spread to lymph nodes, liver, spleen, and other areas and to thus become a systemic cancer. Because of this variable behavior, it is difficult to predict how any individual tumor is going to behave. The variability thus creates uncertainty in deciding what a particular dog's prognosis is, whether staging tests to assess for metastasis are needed, and even what treatments will be necessary for best outcome. In addition to controversies over the potential for development of systemic disease, or diffuse metastasis, controversies also exist over what treatment is needed to best attain local control of these tumors. This article will briefly discuss the diagnosis of MCTs in dogs and will summarize the literature in regards to the controversial topics surrounding the more aggressive form of this disease, with recommendations made based on published studies.
\end{abstract}

Keywords: mitotic index, mastocytosis, tyrosine kinase inhibitor, histologic grade

\section{Introduction}

Mast cell tumors (MCTs) arise from malignantly transformed mast cells. In dogs, most of these tumors arise as primary tumors in the skin. They are the most common skin tumor in dogs, accounting for roughly $20 \%$ of all reported skin tumors. ${ }^{1}$ Any breed may be affected with MCTs, but certain breeds are predisposed, including golden retrievers, Labrador retrievers, Boston terriers, boxers, and pugs. Pugs are more likely to have multiple MCTs at diagnosis ( $56 \%$ of pugs with MCT in one study), but these tumors demonstrate more benign behavior and rarely lead to death. ${ }^{2}$ MCTs can affect dogs of any age but typically affect middle-aged to older dogs. An underlying etiology for most tumors cannot be identified. Breed predilections support some component of underlying genetic causes. Mutations in the c-kit tyrosine kinase receptor, which can lead to malignant transformation of mast cells, are found in $25 \%-30 \%$ of intermediate to high-grade tumors. ${ }^{3,4}$ KIT mutations will be further discussed in regards to both prognosis and treatment options for MCTs.

MCTs can be located anywhere on the body and may lie within the dermis and/or subcutis. They have a wide range of gross appearance, from raised and superficial to very deep and fixed; they may feel soft and fluctuant or firm. Most MCTs are easily diagnosed with fine needle aspiration (FNA). Infrequently, MCT granules will not stain with Diff-Quik (Jorgensen Laboratories Inc., Loveland, CO, USA) and need to be stained with a Wright's stain. On Diff-Quik cytology, if eosinophils are seen along with large round cells that lack granules, suspicion should be raised for an MCT and 
the slide submitted to a clinical pathology laboratory for a non-Diff-Quik stain. ${ }^{5}$

The majority of MCTs will be cured with surgical excision. ${ }^{1}$ Prognostic factors for predicting MCTs that will exhibit a more aggressive biologic behavior - ie, tumors that will not be cured despite local excision and that will ultimately lead to the patient's death - are varied as well as controversial. When to pursue staging tests in dogs with MCTs, which tests to perform, and treatment recommendations beyond surgery are based on the predicted biologic behavior of the tumor, with staging diagnostics and systemic therapy the recommendation for dogs with biologically aggressive MCTs.

\section{Prognostic factors relating to history and physical examination}

Some factors that can be obtained from a history and physical examination that are generally accepted to carry a more guarded prognosis in dogs with MCTs include recent, rapid tumor growth and fixed, ulcerated tumors. ${ }^{6-8}$ Although publications regarding these features are limited, one early study reported doubling of the survival percentage at 30 weeks post-MCT excision for dogs with slow-growing tumors versus (vs) those with more rapidly growing masses. ${ }^{8}$ Biologically, both the ability to grow quickly and to become fixed to deeper tissues are physical manifestations of more aggressive behavior. Tumor location on the body can also be associated with biologic behavior; this topic is more controversial and the pertinent locations and published papers are highlighted as follows.

\section{Mucocutaneous location}

In limited published cases, eyelid margin MCTs appeared to have relatively benign behavior and were effectively treated with local therapy, although one dog was reported to have regional lymph node (LN) metastasis. ${ }^{9-11} \mathrm{MCT}$ of the conjunctiva may be of concern only locally, without reported metastasis in three dogs. ${ }^{12,13}$ In a paper evaluating chemotherapy for high-risk MCT patients, eleven dogs with mucous membrane MCTs (vulva, prepuce, conjunctiva, oral cavity) had significantly shorter median survival times (MST) than 50 dogs with MCTs of haired skin. ${ }^{14}$ However, a recent paper of 32 dogs with 33 conjunctival MCTs treated with surgery alone showed prolonged survival times, with only two dogs having local recurrence despite incomplete margins in 25 cases, and no dogs dying of mast cell-related disease. ${ }^{15}$

\section{Muzzle/perioral/oral location}

Early case reports described aggressive behavior and local metastatic disease at diagnosis in two dogs with MCT of the lip; survival times were 6 months or less. ${ }^{16,17}$ Of five dogs with MCT of the tongue, two presented with LN and/or systemic metastasis, and two of the remaining three had postoperative local recurrence leading to euthanasia. ${ }^{18}$ Larger, more recent studies confirmed that MCTs on the muzzle, perioral mucocutaneous junction, or oral mucosa have a more aggressive biologic behavior, with increased risk of locoregional LN metastasis. ${ }^{19-21}$ The rate of documented metastasis to local (mandibular) LNs was 55\%-59\%, compared with a $<10 \%$ rate for other cutaneous sites. Despite a high rate of metastasis, the MSTs of the dogs were prolonged at 30 months, 52 months, and median not reached. Treatments varied in these cases, with many dogs receiving surgery, radiation, and chemotherapy. Dogs with LN metastasis had significantly shorter MSTs than dogs without nodal metastasis, with medians of 14 months, less than 20 months, and 9 months. ${ }^{19-21}$

\section{Inguinal location}

Historically, the "back half" of the dog was considered to carry a worse prognosis, and although this is generally not supported, there are still concerns about MCTs located in the inguinal areas, especially involving the prepuce or scrotum. One study reported on dogs with MCT in the perineal and/or inguinal region treated intensively, most receiving trimodality therapy with surgery, radiation, and chemotherapy. The MST was 37 months, and the authors concluded that with appropriate therapy dogs with perineal or inguinal MCTs can do well. ${ }^{22}$ It is important to note that these dogs received a more aggressive treatment protocol than do the majority of dogs with MCTs at other cutaneous sites. A later study compared the outcome of dogs with inguinal or perineal MCTs with dogs with MCTs in other cutaneous locations; these dogs received a range of therapies. When the 12 dogs with preputial or scrotal MCTs were analyzed separately from other inguinal or perineal tumors, their disease-free interval was significantly shorter (4.2 months) than for the 84 dogs with tumors in noninguinal cutaneous locations (33.9 months). ${ }^{23}$ Dogs with preputial or scrotal MCTs were also significantly more likely to have received chemotherapy, thus potentially biasing the results. The authors state that the power of the study may not have been adequate to identify a difference between the locations. Scrotal and preputial MCTs may have a more malignant biological behavior; further study is needed.

\section{Subcutaneous location}

Recently, several papers presented information regarding MCTs in the subcutaneous (SQ) location. ${ }^{24-26}$ As the grading 
system was developed only with cutaneous tumors, it cannot necessarily be applied to SQ tumors with prognostic accuracy. Survival times for dogs with the SQ tumors were found to be prolonged, with the majority of dogs in both papers ( 53 dogs and 306 dogs) not succumbing to mast cell disease. ${ }^{24,25}$ Out of the 306 dogs, only $8 \%$ had local recurrence, and only $4 \%$ had metastasis. ${ }^{25}$ Risk factors identified for local recurrence and metastasis included mitotic index (MI), infiltrative vs circumscribed histologic pattern, the presence of multinucleation, as well as Ki67, Ki67 + AgNOR, and KIT cellular localization pattern. ${ }^{25,26}$ As most of the dogs with SQ MCTs were cured, and MI had the strongest correlation with clinical outcomes, there seems to be little need for, or benefit in, assessment of the risk factors that require additional staining.

\section{Histologic information Grade}

A histologic grading scheme (I-III) was developed for classification of MCTs affecting haired skin and is still one of the most accepted prognostic indicators of canine MCT behavior. ${ }^{1}$ However, histologic assessment is prone to operator subjectivity, giving rise to extremely variable grading results for the same tumor among different pathologists. ${ }^{27,28}$ In one study with ten pathologists evaluating the same $60 \mathrm{MCTs}$, there was agreement on grade by all pathologists for only four tumors, and six of the MCTs had all three grades assigned. Differences in the references used to grade the MCTs were the suspected reason for the disparity, with six different references being used. ${ }^{28}$ In a follow-up study involving the same tumors and pathologists, the Patnaik grading scheme $^{29}$ was used by all. Although mean agreement did improve, only 16 of 60 tumors received the same grade by all, and three of 60 tumors still received all three grades. ${ }^{27}$ The subjectivity and variability between pathologists bring into question the heavy reliance placed on the grade of an MCT to predict its behavior. In addition, most MCTs are designated grade II, and most of these are cured with surgery. How to identify the subset of grade II tumors that will show that aggressive behavior is a matter of ongoing debate and study.

To address some of the grading concerns, a two-tier (high vs low grade) grading scheme was proposed in a study of 95 dogs with MCTs treated with surgical excision alone. ${ }^{30}$ High-grade tumors had one of the following criteria: MI of $\geq 7$, three or more multinucleated cells or cells with bizarre nuclei in 10 high-power fields, or karyomegaly. Areas of highest mitoses or anisokaryosis were evaluated. MST for high grade (ten dogs) was 3.6 months vs median not reached
( $>2$ years) for low grade ( $85 \mathrm{dogs})$. A retrospective study with 47 dogs attempted to validate the two-tier scheme; although some of the data reported were inconsistent (including no description of the MI of the cases), the high-grade cases did have significantly worse progression-free and overall survivals than the low-grade ones. ${ }^{31}$ Further studies validating this proposed system are warranted; currently, the author's university pathology department provides the standard grade, an MI, and the two-tier grade for every sample. Clinically, heavy reliance is placed on the MI for behavior prediction.

\section{Other assessments on biopsies - highlighting mitotic index}

As grade is so variable and subjective, many other prognostic factors, including DNA aneuploidy, c-kit-staining pattern, presence of c-kit mutations, microvessel density, Ki67, proliferating cell nuclear antigen, and MI, have been evaluated in an attempt to better predict the behavior of canine MCTs and to pick out the "bad" grade II tumors. Two groups have evaluated the MI (total number of mitotic figures counted in ten high-power fields; fields with the highest mitoses counted) and found that it is predictive of survival time, even within the grade II tumor category. The groups did identify different values for the index, with the first paper showing an MST of $>70$ months for an index score $\leq 5$ and survival $<2$ months for a score $>5 .{ }^{32}$ The second group wrote a letter to the editor in response to the first paper and confirmed that high scores have very short survival times, with index scores broken down into three groups: $\mathrm{MI}=0, \mathrm{MST}$ not reached; $\mathrm{MI}=1-7$, MST $=18$ months; and MI $>7$, MST $=3$ months. ${ }^{33}$ The cutoff of $\mathrm{MI} \geq 7$ was subsequently adopted for the two-tier system.

Although MCT histologic "prognostic panels" are offered by some laboratories, no publications have shown how these panels may provide additional benefit over the MI and grade for prediction of tumor behavior.

\section{Staging information}

In general, staging tests for asymptomatic dogs with cutaneous MCTs are extremely low yield. The test most often positive is regional LN aspiration. Cytologic assessment of the locoregional $\mathrm{LN}$ is important, even if the node is not enlarged. In a study of dogs with muzzle MCT, four of eleven LNs with metastases were of normal size. ${ }^{19}$ Another study with 55 dogs with confirmed LN metastasis and 35 dogs without metastasis showed a sensitivity of $71 \%$ and specificity of $54 \%$ for palpation as a predictor of metastasis. ${ }^{34}$ Sixteen of $35 \mathrm{dogs}$ 
(46\%) with normal size LNs on physical examination had metastasis, whereas in another study eight out of $21(38 \%)$ normal size LNs showed metastasis. ${ }^{35}$ FNA of the LN is ideally performed prior to excision of the primary MCT, as surgical treatment of the tumor can produce confusing LN results due to local postoperative inflammation. As mast cells can be a normal feature in LNs, some MCT cases will not have a definitive answer on LN cytology. Criteria for LN involvement have been proposed and used in several subsequent reports. ${ }^{34-37}$ Prognostically, the implication of a metastatic LN is also a controversial topic. LN involvement has been associated with a worse prognosis in a number of studies..$^{20,36-39}$ However, several papers also report long-term survival in dogs with LN involvement where the primary tumor and the $\mathrm{LN}$ are treated to achieve local control using surgery with or without radiation therapy. ${ }^{14,34,35,40,41}$ Chemotherapy was used in many of the reported cases as well, although the protocols varied, and the added benefit of chemotherapy after local control was achieved cannot be proven via the retrospective noncontrolled studies that currently exist. To summarize the information in the literature, LN involvement may carry a worse prognosis, yet dogs can still have prolonged survival with adequate treatment of the primary tumor and the metastatic node, with chemotherapy potentially having a benefit as well. Assessment of any locoregional LNs, whether normal sized or enlarged, with cytology or histopathology is critical to determine the stage of the tumor and appropriate therapy for the patient.

Other staging tests are rarely positive and may have false positive results as well. In general, staging tests other than LN aspiration are recommended in patients who have negative prognostic factors associated with their MCT. Thoracic radiographs are indicated to evaluate the sternal LN if the mass is on the ventral abdomen, or to rule out other nonMCT diseases. MCTs metastasize so rarely to the lungs that radiographs are not indicated to evaluate for pulmonary spread. Buffy coat preparation to look for circulating mast cells is a quick and easy test, but it is both insensitive and nonspecific. The bone marrow may still be infiltrated in spite of a normal buffy coat, and dogs with skin disease, parvo virus, and nonmast cell illnesses often have positive buffy coats despite not having an $\mathrm{MCT}^{42-44}$ Bone marrow evaluation for mast cell infiltration is positive in $<5 \%$ of cases, even in cases with poorly differentiated tumors, and thus is not a staging test that is recommended as standard. ${ }^{45}$

Abdominal ultrasound (US) also rarely finds evidence of MCT metastasis, although US is needed to evaluate the sublumbar LNs if the MCT is on the back half of the patient.
The benefit of FNA and cytology of ultrasonographically normal-appearing liver and spleen for staging of dogs with MCTs is another topic of ongoing debate. Mast cells can be found in moderate numbers in normal canine liver and spleen, and although increased numbers were found in splenic aspirates in 51 MCT-bearing dogs with normal spleens on US compared with 32 unaffected dogs, the splenic cytology did not correlate with systemic behavior. ${ }^{46}$ In another study, 52 dogs with MCTs underwent ultrasound evaluation and FNA with cytology of their liver and spleen. ${ }^{47}$ Cytologic criteria of MCT infiltration included clustering of well-differentiated mast cells, large numbers of well-differentiated mast cells, or mast cells with atypical morphology (pleomorphic and poorly granulated). The dogs were separated into two groups: those without MCT infiltration into either organ $(n=42)$ and those with infiltration into one or both organs $(n=10)$; survival time between these groups was significantly different at 733 days vs 34 days, respectively $(P<0.0001)$. This dramatic difference in survival time supports that the cytologic findings were consistent with systemic MCT. Survival time based on US appearance alone in the dogs was not significant, suggesting that cytologic evaluation of the spleen and liver may be indicated for complete staging of dogs with MCT regardless of the ultrasonographic findings. ${ }^{47}$ More recently, 19 dogs with clinically aggressive grade II or III MCTs treated with vinblastine/lomustine chemotherapy were evaluated to determine the specificity and sensitivity of US findings in determination of MCT infiltration of the liver and/or spleen, using cytologic assessment to determine infiltration. ${ }^{48}$ Seven dogs had MCT infiltration, and the sensitivity of US for detection of infiltration was $43 \%$ for the spleen and $0 \%$ for the liver. Dogs with cytologic determination of infiltration of the spleen or liver had significantly shorter survival than dogs without infiltration (100 days vs 291 days and 100 days vs 276 days, respectively, $P<0.0001$ ), this clinical survival thus corroborating the cytologic finding of systemic disease. Due to the poor sensitivity of US in detection of cytologically confirmed organ infiltration and the decreased survival associated with identified infiltration, FNA and cytology of the spleen and liver are recommended for staging of dogs with MCT thought to be at high risk for metastasis. ${ }^{48}$

\section{What is needed for local control? Surgical margins}

Although surgery is the mainstay of therapy for MCTs, and is curative in a large percentage of cases, there is still much controversy over surgical margins. Removing the tumor with gross margins of $2-3 \mathrm{~cm}$ lateral margins and a deep margin one tissue 
plane beyond the tumor has been recommended. Twenty-three MCTs in 21 dogs were evaluated for completeness of excision at $1 \mathrm{~cm}, 2 \mathrm{~cm}$, and $3 \mathrm{~cm}$ from the tumor edge; $100 \%$ of tumors had complete excision at the $2 \mathrm{~cm}$ margin mark. Only grade I (three) and grade II (20) MCTs were in the study, so it is not clear whether $2 \mathrm{~cm}$ margins will be adequate for grade III tumors. ${ }^{49}$ In a subsequent study, 91\% (21/23) of grade I and II tumors were completely excised with $2 \mathrm{~cm}$ margins and had no local recurrence for $>538$ days. ${ }^{50}$ Thus, $2 \mathrm{~cm}$ margins provide good results for grade I and II MCTs, with decreased morbidity. A recent publication described 40 dogs with cutaneous $(87 \%)$ and SQ (13\%) MCTs treated with a modified proportional margin approach. ${ }^{51}$ Tumors were resected with a lateral margin equal to their widest diameter, with a maximum lateral margin of $4 \mathrm{~cm}$, and with a minimum depth of one fascial plane deep to the tumor. Gross surgical margins obtained ranged from $5 \mathrm{~mm}$ to $40 \mathrm{~mm}$. Tumors ranged in grade and included $51 \%$ grade I, 44\% grade II, and 5\% grade II; with the two-tier system $90 \%$ were low grade and $10 \%$ high grade. Complete excision was obtained in $85 \%$ of tumors, whereas $15 \%$ had dirty margins. Only one tumor regrew locally; the median follow-up time for the dogs was 420 days. This novel approach to MCT surgical margin excision with individual tailoring for the patient appears to provide good outcomes; validating studies are warranted.

\section{Histologic margins}

Another controversy rages over what to do about clean vs dirty surgical margins. One difficulty is that no consensus exists as to how to define completeness of excision. A report on 100 dogs with 115 completely resected MCTs showed no recurrence or metastasis in grade I and II tumors when excised with histologic lateral margins $\geq 10 \mathrm{~mm}$ and deep margins $\geq 4 \mathrm{~mm} .{ }^{52}$ This paper was attempting to address how wide a gross margin needs to be for complete tumor excision, but as only histologic margins were measured, it speaks more to the issue of what size a microscopic margin size can be for local control. However, MCTs will recur with both clean and dirty margins, and the percentage of such recurrence varies widely between papers. With recurrence defined as a new mass within $2 \mathrm{~cm}$ of a previous incision developing up to 1 year postoperatively, two papers stated a $19 \%-37.5 \%$ recurrence with clean margins. ${ }^{53,54}$ Other papers cite a lower recurrence with clean margins, from $0 \%$ to $11 \%{ }^{52,55,56,57}$ With incomplete resection, one paper found a $63.6 \%$ recurrence rate. ${ }^{54}$ This is in contrast to a number of papers that report a much lower recurrence rate for dirty margins, generally ranging from $12 \%$ to $30 \% .^{25,55,58-61}$ In one of these papers, only $23 \%$ of $28 \mathrm{dogs}$ with incompletely resected grade II tumors experienced local regrowth. The combination of Ki67 and proliferating cell nuclear antigen scores was prognostic for local recurrence, and dogs with such recurrence had significantly decreased survival times. ${ }^{59}$ Thus, in cases where further local therapy is being discussed, these proliferation indices may be beneficial. Also, although a rare event, SQ MCT recurrence was associated with a number of histologic markers (see section on subcutaneous location). A recent study further highlighted the difficulties with MCT margins; this study evaluated the two-tier histologic grade as a predictor for regrowth in cases with clean margins. ${ }^{62}$ Lowgrade tumors were found to not recur despite narrow $(29 \%$ $\leq 3 \mathrm{~mm}$ ) clean microscopic margins, but high-grade tumors with clean margins recurred frequently $(35.9 \%)$ but with no relation to the width of the tumor-free margin, thus showing prediction of regrowth to be impossible based on evaluation of the size of the clean margin. This rate of local regrowth for completely excised tumors is surprisingly high, higher than most reports of regrowth with incomplete resections. This rate may, in part, be due to the large percentage (43\%) of tumors in the study that were high grade. Lastly, looking at addressing further therapy for dirty or close margins, a recent retrospective study compared survival and local recurrence in dogs with incompletely or narrowly $(<3 \mathrm{~mm}$ margin) resected MCTs treated with primary re-excision or radiation therapy vs no additional local therapy. ${ }^{63}$ Recurrence was noted in $38 \%$ of the no additional local treatment group, vs $13 \%$ in the primary re-excision group and $8 \%$ in the radiation group. Survival times for the groups receiving further local therapy were significantly longer than for the group that did not. This study lends support to the benefit of further local therapy for incompletely or narrowly resected MCTs, while also confirming that despite dirty or close margins less than $40 \%$ of tumors recur. ${ }^{63}$ With reported low recurrence rates for "incomplete" resections, and variable regrowth despite "clean" margins, more aggressive surgery or radiation therapy to treat any potential "residual" local disease becomes a controversial topic and one for further discussion with pet owners. More information is needed in regards to histologic margin assessment for MCTs in order to interpret clean vs dirty margin results and to make logical and substantiated treatment recommendations for owners.

\section{Radiation therapy}

MCTs are very radiosensitive, and multiple reports show that fractionated radiation (daily or every other day for 15-18 treatments with total doses from 46 gray to 54 gray) is 
an effective adjunctive therapy for incomplete margins after surgery, with $75 \%-96 \%$ of dogs having a local cure (no evidence of regrowth at 3-5 years postradiation). ${ }^{40,64-69}$ However, the controversies over what "incomplete" margins mean, and how likely such dirty margins are to lead to tumor regrowth, bring into question the necessity of radiation for some cases. If the tumor is in a location where only minimal resection can be obtained grossly, then radiation is offered as a follow-up therapy in the author's practice. Radiation can also be very beneficial when used in large fraction weekly doses for four to six treatments to treat bulky, nonsurgical MCTs. ${ }^{69}$ Combining large fraction radiation with toceranib phosphate (see section on systemic therapy) was tolerated and showed a local response rate of $76 \%$; MST was not reached with a median follow-up time of 374 days. ${ }^{70}$ This combination therapy may be a good treatment option for nonsurgical MCTs.

\section{When and what to use for systemic therapy}

Additional therapy (chemotherapy, tyrosine kinase inhibitor [TKI] therapy) may be considered in dogs with poor prognostic indicators (eg, grade III, high MI, poor location, metastasis) after excision of the MCT. Dogs with nonexcisable tumors may also be considered for chemotherapy or TKI therapy; in general, chemotherapy is more successful against microscopic disease but can be beneficial in cases with bulky tumors.

Vinblastine/prednisone and lomustine are commonly used chemotherapeutics for MCTs. Few controlled studies have been published that look at the response rate to these drugs. Most of the literature consists of retrospective studies that include a range of MCT grades and stages with chemotherapy protocols that are not standardized. Nevertheless, there are enough reports of effect against bulky disease, with response rates ranging from $11 \%$ to $57 \%$, to support the use of these chemotherapy drugs for MCTs. ${ }^{7,14,71-74}$ Many chemotherapy protocols exist; a common protocol for vinblastine/prednisone is shown in Figure 1. This eight-dose protocol is to treat dogs with aggressive MCT without evidence of gross disease. If measurable disease is present, then the treatment is based on effect - with continued weekly doses until maximum response, then every 2 weeks, and then decreasing the frequency of administration to as infrequent as possible to still maintain tumor control. At the author's practice, as long as it is well tolerated (no grade III or IV toxicities), ${ }^{75}$ vinblastine is dose escalated by $0.25 \mathrm{mg} / \mathrm{m}^{2}$ to a maximum dose of $3.5 \mathrm{mg} / \mathrm{m}^{2} .^{76}$ Lomustine as a single agent is dosed at $60-90 \mathrm{mg} / \mathrm{m}^{2}$ orally every 2-3 weeks. Lomustine can be very myelosuppressive, as well as hepatotoxic. ${ }^{77}$ Liver enzymes need to be monitored along with routine complete blood counts. Daily Denamarin ${ }^{\circledR}$ (Nutramax Laboratories Veterinary Sciences, Inc., Lancaster, $\mathrm{SC}, \mathrm{USA}$ ) is recommended while dogs are receiving lomustine. ${ }^{78}$ Recently, a study evaluating water-soluble micellar paclitaxel (Paccal Vet ${ }^{\circledR}$; Abbott Animal Health, Abbott Park, IL, USA) for treatment of nonresectable grade II or III MCTs in dogs showed Paccal Vet ${ }^{\circledR}$ to be safer and more efficacious (30\% biologic response rate) than lomustine (11\% biologic response rate). ${ }^{74}$ This product will soon be available in the United States under conditional approval, with label indications for treatment of mammary tumors and squamous cell carcinoma; hopefully, full approval will follow shortly, allowing MCTs to be treated via off-label use.

Masitinib and toceranib phosphate, orally administered small molecule inhibitors of TKs, have activity against bulky canine MCTs. These drugs are likely to also provide benefit in the microscopic disease setting, including treatment in dogs with MCTs with poor prognostic factors such as high grade, high MI, and poor location. Masitinib (Kinavet ${ }^{\circledR}$ or Masivet $^{\circledR}$; AB Science, Chatham, NJ, USA) is a TKI that mainly works against KIT. A publication described $202 \mathrm{dogs}$ with grade II or III MCTs in a randomized study evaluating masitinib. ${ }^{79}$ Dogs treated with masitinib at $12.5 \mathrm{mg} / \mathrm{kg}$ orally once daily had a longer time to disease progression (178 days) vs dogs receiving placebo (75 days). Masitinib did not improve overall survival time compared with placebo, except in the subset of dogs with KIT mutations. In that group, survival with masitinib was 417 days vs 182 days with placebo. ${ }^{79}$ A subsequent paper examined the 12 month and 24 month survival rates in 132 dogs that continued on their respective treatments (either placebo or masitinib) from the earlier study; these treatments were continued until disease progression. ${ }^{80}$ Survival rates at 12 months and 24 months were significantly improved in the dogs receiving masitinib, with $62 \%$ and $39.8 \%$ of masitinib dogs alive at 12 months and 24 months, vs $36 \%$ and $15 \%$ of placebo dogs alive at the same time points. However, the MSTs of 617 days for masitinib dogs were not significantly different from the median of 322 days for placebo. Currently, in the United States, masitinib is conditionally approved and thus can legally be used only for the label indications ("For the treatment of nonresectable grade II or III cutaneous MCTs in dogs that have not previously received radiotherapy and/or chemotherapy except corticosteroids"). Masitinib is fully approved in Europe. Generally well tolerated, monitoring of patients on masitinib includes complete blood count, chemistry panels, and urinalysis initially every 2 weeks for two to three visits 


\section{Date Dose Weight $/ \mathrm{m}^{2}$}

$\begin{array}{lll}\text { Week } 1 & \text { Vinblastine } 2 \mathrm{mg} / \mathrm{m}^{2} \text { IV } & \text { Prednisone } 2 \mathrm{mg} / \mathrm{kg} \text { PO SID } \\ \text { Week } 2 & \text { Vinblastine } 2 \mathrm{mg} / \mathrm{m}^{2} \text { IV } & \\ & \text { Prednisone } 1.5 \mathrm{mg} / \mathrm{kg} \text { PO SID } & - \\ \text { Week } 3 & \text { Vinblastine } 2 \mathrm{mg} / \mathrm{m}^{2} \mathrm{IV} & - \\ \text { Week } 4 & \text { Vinblastine } 2 \mathrm{mg} / \mathrm{m}^{2} \text { IV } & - \\ \text { Week } 12 & \text { Vinblastine } 2 \mathrm{mg} / \mathrm{m}^{2} \text { IV } & - \\ \text { Week } 10 & \text { Vinblastine } 2 \mathrm{mg} / \mathrm{m}^{2} \text { IV } & -\end{array}$

A CBC must be performed within 24 hours prior to chemotherapy administration

Delay chemotherapy by 3-7 days and recheck $C B C$ if:

Neutrophil count $<2,000$ cells $/ \mu \mathrm{L}$

Platelet count $<50,000$ cells $/ \mu \mathrm{L}$

Patient is exhibiting GI signs secondary to previous chemotherapy

Figure I Canine mast cell tumor protocol.

Abbreviations: CBC, complete blood count; GI, gastrointestinal; IV, intravenous, PO, orally; SID, once a day.

and then monthly thereafter. Severe hypoproteinemia with protein loss in the urine is a very serious, although rare, side effect; thus, monitoring of the urine is critical. If proteinuria is noted, the drug should be discontinued.

Toceranib phosphate (Palladia ${ }^{\circledR}$; Pfizer, Inc., New York, NY, USA) has activity against the split kinase family members (vascular endothelial growth factor receptor, platelet-derived growth factor receptor, and KIT) and is believed to have both direct antitumor and antiangiogenic activity. Toceranib phosphate is the first anticancer drug approved by the US
Food and Drug Administration for veterinary use. An early study showed $54 \%$ of dogs with a variety of tumors, including sarcoma, carcinoma, myeloma, melanoma, and MCTs, having some response to the drug. ${ }^{81}$ A subsequent study in 149 dogs with bulky (nonresectable) grade II or III MCTs showed a $37 \%$ response rate in dogs receiving toceranib phosphate $(3.25$ $\mathrm{mg} / \mathrm{kg}$ orally every other day) $(\mathrm{n}=86)$ vs an $8 \%$ response in dogs treated with placebo $(n=63) .{ }^{82}$ After 6 weeks, the study was unblinded and all dogs were eligible to receive toceranib phosphate. In the 58 placebo dogs that subsequently received 
toceranib phosphate, $40 \%$ showed a response. The overall response rate of $145 \mathrm{dogs}$ that received toceranib phosphate was thus $43 \%$ ( 21 complete responses, 41 partial responses). If dogs that maintained stable disease for $\geq 10$ weeks (12\%) are considered to have shown a biological response to toceranib phosphate, then the overall response rate increased to $59.5 \%$. Although dogs with KIT mutations were more likely to have a biological response (82\%), dogs without mutations still showed a response (54\%). Thus, the author does not routinely test for KIT mutations prior to toceranib phosphate therapy. If bulky mast cell disease is present, response to therapy will guide the therapy, as KIT mutation status does not guarantee or rule out potential toceranib phosphate response. Although initial studies showed significant gastrointestinal toxicity, a recent report evaluating lower doses of toceranib phosphate showed good efficacy and good tolerability with dosages of $2.5-2.9 \mathrm{mg} / \mathrm{kg}$ every other day. ${ }^{83}$ Monitoring of patients on toceranib phosphate is similar to that for masitinib. Toceranib phosphate can lead to milder protein-losing nephropathy than is seen with masitinib; if noted, toceranib phosphate should be discontinued. Most cases respond well and quickly to enalapril, and reinitiation of toceranib phosphate can be considered.

Recent studies and ongoing investigations are looking at combining toceranib phosphate with chemotherapy. ${ }^{84,85}$ For dogs with MCTs, vinblastine at $1.6 \mathrm{mg} / \mathrm{m}^{2}$ every other week combined with toceranib phosphate $3.25 \mathrm{mg} / \mathrm{kg}$ orally every other day was safe and showed a $71 \%$ objective response rate. ${ }^{85}$ The study authors recommend a prospective randomized trial of this combination compared with single-agent therapy to confirm the results. Additionally, it would be beneficial to know how the currently recommended lower dose of toceranib phosphate will work in combination.

In summary, many options for systemic therapy exist for dogs with aggressive MCTs. Frequency of administration, necessity for travel to the veterinary clinic, side effect profiles, cost, and drug approval status all play a role in discussions with pet owners and subsequent choices made. A course of chemotherapy may be followed by long-term use of toceranib phosphate. If masitinib is to be used in the United States, then it must be the first-line therapy per label indications, due to conditional approval in the United States. Multiple different chemotherapy protocols, including the use of TKIs, may all be used during the treatment efforts against macroscopic MCTs.

\section{Ancillary therapy}

Histamine blockers are used preoperatively with large masses, or for life with nonresectable masses or systemic disease.
Famotidine (H2 blocker) at $0.5 \mathrm{mg} / \mathrm{kg}$ once daily can help to prevent/treat gastric ulcers, and diphenhydramine (H1 blocker) at $2 \mathrm{mg} / \mathrm{kg}$ three times daily is used to prevent systemic allergic reactions/anaphylaxis.

\section{Conclusion}

MCTs are challenging tumors to treat and make a prognosis for, based on their widely variable biologic behavior. Most MCTs will be cured with appropriate local therapy. Bearing in mind the ongoing controversies discussed, the use of predictive factors highlighted in this article can help to determine which tumors are more likely to become a life-limiting issue for a canine patient, thus guiding recommendations regarding pursuit of further testing and therapies.

\section{Disclosure}

The author is a member of a consulting panel of veterinarians for Zoetis and their product Palladia ${ }^{\circledR}$ (toceranib phosphate).

\section{References}

1. London C, Thamm D. Mast Cell Tumors. 5th ed. St Louis, MO: Saunders Elsevier; 2013.

2. McNiel EA, Prink AL, O'Brien TD. Evaluation of risk and clinical outcome of mast cell tumours in pug dogs. Vet Comp Oncol. 2006;4(1):2-8.

3. Downing S, Chien MB, Kass PH, Moore PE, London CA. Prevalence and importance of internal tandem duplications in exons 11 and 12 of c-kit in mast cell tumors of dogs. Am J Vet Res. 2002;63(12):1718-1723.

4. Letard S, Yang Y, Hanssens K, et al. Gain-of-function mutations in the extracellular domain of KIT are common in canine mast cell tumors. Mol Cancer Res. 2008;6(7):1137-1145.

5. Jackson DE, Selting KA, Spoor MS, Henry CJ, Wiedmeyer CE. Evaluation of fixation time using Diff-Quik for staining of canine mast cell tumor aspirates. Vet Clin Pathol. 2013;42(1):99-102.

6. Mullins MN, Dernell WS, Withrow SJ, Ehrhart EJ, Thamm DH, Lana SE. Evaluation of prognostic factors associated with outcome in dogs with multiple cutaneous mast cell tumors treated with surgery with and without adjuvant treatment: 54 cases (1998-2004). J Am Vet Med Assoc. 2006;228(1):91-95.

7. Thamm DH, Mauldin EA, Vail DM. Prednisone and vinblastine chemotherapy for canine mast cell tumor - 41 cases (1992-1997). J Vet Intern Med. 1999;13(5):491-497.

8. Bostock DE. The prognosis following surgical removal of mastocytomas in dogs. J Small Anim Pract. 1973;14(1):27-41.

9. Northrup NC, Roberts RE, Harrell TW, Allen KL, Howerth EW, Gieger TL. Iridium-192 interstitial brachytherapy as adjunctive treatment for canine cutaneous mast cell tumors. J Am Anim Hosp Assoc. 2004;40(4):309-315.

10. Roberts SM, Severin GA, Lavach JD. Prevalence and treatment of palpebral neoplasms in the dog: 200 cases (1975-1983). J Am Vet Med Assoc. 1986;189(10):1355-1359.

11. Jacobi S, Stanley BJ, Petersen-Jones S, Dervisis N, Dominguez PA. Use of an axial pattern flap and nictitans to reconstruct medial eyelids and canthus in a dog. Vet Ophthalmol. 2008;11(6):395-400.

12. Barsotti G, Marchetti V, Abramo F. Primary conjunctival mast cell tumor in a Labrador Retriever. Vet Ophthalmol. 2007;10(1):60-64.

13. Johnson BW, Brightman AH, Whiteley HE. Conjunctival mast cell tumors in two dogs. J Am Anim Hosp Assoc. 1988;24:439-442. 
14. Thamm DH, Turek MM, Vail DM. Outcome and prognostic factors following adjuvant prednisone/vinblastine chemotherapy for highrisk canine mast cell tumour: 61 cases. J Vet Med Sci. 2006;68(6): 581-587.

15. Fife M, Blocker T, Fife T, Dubielzig RR, Dunn K. Canine conjunctival mast cell tumors: a retrospective study. Vet Ophthalmol. 2011;14(3):153-160.

16. Herman LH, Slaughter LJ, Martin DP. Malignant mastocytoma in a dog. J Am Vet Med Assoc. 1967;151(10):1322-1324.

17. Patnaik AK, MacEwen EG, Black AP, Luckow S. Extracutaneous mast-cell tumor in the dog. Vet Pathol. 1982;19(6):608-615.

18. Beck ER, Withrow SJ, McChesney AE, et al. Canine tongue tumors: a retrospective review of 57 cases. J Am Anim Hosp Assoc. 1986;22(4):525-532.

19. Gieger TL, Theon AP, Werner JA, McEntee MC, Rassnick KM, DeCock HE. Biologic behavior and prognostic factors for mast cell tumors of the canine muzzle: 24 cases (1990-2001). J Vet Intern Med. 2003;17(5):687-692

20. Hillman LA, Garrett LD, de Lorimier LP, Charney SC, Borst LB, Fan TM. Biological behavior of oral and perioral mast cell tumors in dogs: 44 cases (1996-2006). J Am Vet Med Assoc. 2010;237(8):936-942.

21. Elliott JW, Cripps P, Blackwood L, Berlato D, Murphy S, Grant IA. Canine oral mucosal mast cell tumours. Vet Comp Oncol. Epub November 11, 2013.

22. Cahalane AK, Payne S, Barber LG, et al. Prognostic factors for survival of dogs with inguinal and perineal mast cell tumors treated surgically with or without adjunctive treatment: 68 cases (1994-2002). J Am Vet Med Assoc. 2004;225(3):401-408.

23. Sfiligoi G, Rassnick KM, Scarlett JM, Northrup NC, Gieger TL. Outcome of dogs with mast cell tumors in the inguinal or perineal region versus other cutaneous locations: 124 cases (1990-2001). J Am Vet Med Assoc. 2005;226(8):1368-1374.

24. Newman SJ, Mrkonjich L, Walker KK, Rohrbach BW. Canine subcutaneous mast cell tumour: diagnosis and prognosis. J Comp Pathol. 2007;136(4):231-239.

25. Thompson JJ, Pearl DL, Yager JA, Best SJ, Coomber BL, Foster RA. Canine subcutaneous mast cell tumor: characterization and prognostic indices. Vet Pathol. 2011;48(1):156-168.

26. Thompson JJ, Yager JA, Best SJ, et al. Canine subcutaneous mast cell tumors: cellular proliferation and KIT expression as prognostic indices. Vet Pathol. 2011;48(1):169-181.

27. Northrup NC, Howerth EW, Harmon BG, et al. Variation among pathologists in the histologic grading of canine cutaneous mast cell tumors with uniform use of a single grading reference. J Vet Diagn Invest. 2005;17(6):561-564.

28. Northrup NC, Harmon BG, Gieger TL, et al. Variation among pathologists in histologic grading of canine cutaneous mast cell tumors. J Vet Diagn Invest. 2005;17(3):245-248.

29. Patnaik AK, Ehler WJ, MacEwen EG. Canine cutaneous mast cell tumor: morphologic grading and survival time in 83 dogs. Vet Pathol. 1984;21(5):469-474.

30. Kiupel M, Webster JD, Bailey KL, et al. Proposal of a 2-tier histologic grading system for canine cutaneous mast cell tumors to more accurately predict biological behavior. Vet Pathol. 2011;48(1):147-155.

31. Takeuchi Y, Fujino Y, Watanabe M, et al. Validation of the prognostic value of histopathological grading or c-kit mutation in canine cutaneous mast cell tumours: a retrospective cohort study. Vet J. 2013;196(3): 492-498.

32. Romansik EM, Reilly CM, Kass PH, Moore PF, London CA. Mitotic index is predictive for survival for canine cutaneous mast cell tumors. Vet Pathol. 2007;44(3):335-341.

33. Elston L, Sueiro FA, Cavalcanti J, Metze K. The importance of the mitotic index as a prognostic factor for canine cutaneous mast cell tumors: a validation study. Vet Pathol. 2009;46(2):362-364.

34. Baginski H, Davis G, Bastian RP. The prognostic value of lymph node metastasis with grade 2 MCTs in dogs: 55 cases (2001-2010). J Am Anim Hosp Assoc. 2014;50(2):89-95.
35. Lejeune A, Skorupski K, Frazier S, et al. Aggressive local therapy combined with systemic chemotherapy provides long-term control in grade II stage 2 canine mast cell tumour: 21 cases (1999-2012). Vet Comp Oncol. Epub May 31, 2013.

36. Krick EL, Billings AP, Shofer FS, Watanabe S, Sorenmo KU. Cytological lymph node evaluation in dogs with mast cell tumours: association with grade and survival. Vet Comp Oncol. 2009;7(2):130-138.

37. Hume CT, Kiupel M, Rigatti L, Shofer FS, Skorupski KA, Sorenmo KU. Outcomes of dogs with grade 3 mast cell tumors: 43 cases (1997-2007). J Am Anim Hosp Assoc. 2011;47(1):37-44.

38. Marconato L, Marchetti V, Francione D, et al. Morphometrical approach for predicting regional lymph node micrometastatic load in canine mast cell tumours: preliminary results. Vet Comp Oncol. 2008;6(3):162-170.

39. Murphy S, Sparkes AH, Blunden AS, Brearley MJ, Smith KC. Effects of stage and number of tumours on prognosis of dogs with cutaneous mast cell tumours. Vet Rec. 2006;158(9):287-291.

40. Chaffin K, Thrall DE. Results of radiation therapy in 19 dogs with cutaneous mast cell tumor and regional lymph node metastasis. Vet Radiol Ultrasound. 2002;43(4):392-395.

41. Rassnick KM, Bailey DB, Russell DS, et al. A phase II study to evaluate the toxicity and efficacy of alternating CCNU and high-dose vinblastine and prednisone (CVP) for treatment of dogs with high-grade, metastatic or nonresectable mast cell tumours. Vet Comp Oncol. 2010;8(2): 138-152.

42. Cayatte SM, McManus PM, Miller WH, Scott DW. Identification of mast cells in buffy coat preparations from dogs with inflammatory skin diseases. J Am Vet Med Assoc. 1995;206(3):325-326.

43. McManus PM. Frequency and severity of mastocytemia in dogs with and without mast cell tumors: 120 cases (1995-1997). J Am Vet Med Assoc. 1999;215(3):355-357.

44. Stockham SL, Basel DL, Schmidt DA. Mastocytemia in dogs with acute inflammatory diseases. Vet Clin Pathol. 1986;15(1):16-21.

45. Endicott MM, Charney SC, McKnight JA, Loar AS, Barger AM, Bergman PJ. Clinicopathological findings and results of bone marrow aspiration in dogs with cutaneous mast cell tumours: 157 cases (1999-2002). Vet Comp Oncol. 2007;5(1):31-37.

46. Finora K, Leibman NF, Fettman MJ, Powers BE, Hackett TA, Withrow SJ. Cytological comparison of fine-needle aspirates of liver and spleen of normal dogs and of dogs with cutaneous mast cell tumours and an ultrasonographically normal appearing liver and spleen. Vet Comp Oncol. 2006;4(3):178-183.

47. Stefanello D, Valenti P, Faverzani S, et al. Ultrasound-guided cytology of spleen and liver: a prognostic tool in canine cutaneous mast cell tumor. J Vet Intern Med. 2009;23(5):1051-1057.

48. Book AP, Fidel J, Wills T, Bryan J, Sellon R, Mattoon J. Correlation of ultrasound findings, liver and spleen cytology, and prognosis in the clinical staging of high metastatic risk canine mast cell tumors. Vet Radiol Ultrasound. 2011;52(5):548-554.

49. Simpson AM, Ludwig LL, Newman SJ, Bergman PJ, Hottinger HA, Patnaik AK. Evaluation of surgical margins required for complete excision of cutaneous mast cell tumors in dogs. J Am Vet Med Assoc. 2004;224(2):236-240.

50. Fulcher RP, Ludwig LL, Bergman PJ, Newman SJ, Simpson AM, Patnaik AK. Evaluation of a two-centimeter lateral surgical margin for excision of grade I and grade II cutaneous mast cell tumors in dogs. J Am Vet Med Assoc. 2006;228(2):210-215.

51. Pratschke KM, Atherton MJ, Sillito JA, Lamm CG. Evaluation of a modified proportional margins approach for surgical resection of mast cell tumors in dogs: 40 cases (2008-2012). J Am Vet Med Assoc. 2013;243(10):1436-1441.

52. Schultheiss PC, Gardiner DW, Rao S, Olea-Popelka F, Tuohy JL. Association of histologic tumor characteristics and size of surgical margins with clinical outcome after surgical removal of cutaneous mast cell tumors in dogs. J Am Vet Med Assoc. 2011;238(11):1464-1469.

53. Jaffe MH, Hosgood G, Kerwin SC, Hedlund CS, Taylor HW. Deionised water as an adjunct to surgery for the treatment of canine cutaneous mast cell tumours. J Small Anim Pract. 2000;41(1):7-11. 
54. Grier RL, Di Guardo G, Myers R, Merkley DF. Mast cell tumour destruction in dogs by hypotonic solution. J Small Anim Pract. 1995;36(9):385-388.

55. Michels GM, Knapp DW, DeNicola DB, Glickman N, Bonney P. Prognosis following surgical excision of canine cutaneous mast cell tumors with histopathologically tumor-free versus nontumor-free margins: a retrospective study of 31 cases. J Am Anim Hosp Assoc. 2002;38(5):458-466.

56. Seguin B, Leibman NF, Bregazzi VS, et al. Clinical outcome of dogs with grade-II mast cell tumors treated with surgery alone: 55 cases (1996-1999). J Am Vet Med Assoc. 2001;218(7):1120-1123.

57. Weisse C, Shofer FS, Sorenmo K. Recurrence rates and sites for grade II canine cutaneous mast cell tumors following complete surgical excision. J Am Anim Hosp Assoc. 2002;38(1):71-73.

58. Misdorp W. Incomplete surgery, local immunostimulation, and recurrence of some tumour types in dogs and cats. Vet Q. 1987;9(3):279-286.

59. Seguin B, Besancon MF, McCallan JL, et al. Recurrence rate, clinical outcome, and cellular proliferation indices as prognostic indicators after incomplete surgical excision of cutaneous grade II mast cell tumors: 28 dogs (1994-2002). J Vet Intern Med. 2006;20(4):933-940.

60. Brocks BA, Neyens IJ, Teske E, Kirpensteijn J. Hypotonic water as adjuvant therapy for incompletely resected canine mast cell tumors: a randomized, double-blind, placebo-controlled study. Vet Surg. 2008;37(5):472-478.

61. Murphy S, Sparkes AH, Smith KC, Blunden AS, Brearley MJ. Relationships between the histological grade of cutaneous mast cell tumours in dogs, their survival and the efficacy of surgical resection. Vet Rec. 2004;154(24):743-746.

62. Donnelly L, Mullin C, Balko J, et al. Evaluation of histological grade and histologically tumour-free margins as predictors of local recurrence in completely excised canine mast cell tumours. Vet Comp Oncol. Epub March 4, 2013.

63. Kry KL, Boston SE. Additional local therapy with primary re-excision or radiation therapy improves survival and local control after incomplete or close surgical excision of mast cell tumors in dogs. Vet Surg. Epub January 6, 2014.

64. Frimberger AE, Moore AS, LaRue SM, Gliatto JM, Bengtson AE. Radiotherapy of incompletely resected, moderately differentiated mast cell tumors in the dog: 37 cases (1989-1993). J Am Anim Hosp Assoc. 1997;33(4):320-324.

65. LaDue T, Price GS, Dodge R, Page RL, Thrall DE. Radiation therapy for incompletely resected canine mast cell tumors. Vet Radiol Ultrasound. 1998;39(1):57-62

66. Hahn KA, King GK, Carreras JK. Efficacy of radiation therapy for incompletely resected grade-III mast cell tumors in dogs: 31 cases (1987-1998). J Am Vet Med Assoc. 2004;224(1):79-82.

67. Poirier VJ, Adams WM, Forrest LJ, Green EM, Dubielzig RR, Vail DM. Radiation therapy for incompletely excised grade II canine mast cell tumors. J Am Anim Hosp Assoc. 2006;42(6):430-434.

68. al-Sarraf R, Mauldin GN, Patnaik AK, Meleo KA. A prospective study of radiation therapy for the treatment of grade 2 mast cell tumors in 32 dogs. J Vet Intern Med. 1996;10(6):376-378.

69. Dobson J, Cohen S, Gould S. Treatment of canine mast cell tumours with prednisolone and radiotherapy. Vet Comp Oncol. 2004;2(3):132-141.

70. Carlsten KS, London CA, Haney S, Burnett R, Avery AC, Thamm DH. Multicenter prospective trial of hypofractionated radiation treatment, toceranib, and prednisone for measurable canine mast cell tumors. J Vet Intern Med. 2012;26(1):135-141.

Veterinary Medicine: Research and Reports

\section{Publish your work in this journal}

Veterinary Medicine: Research and Reports is an international, peer-reviewed, open access journal publishing original research, case reports, editorials, reviews and commentaries on all areas of veterinary medicine. The manuscript management system is completely online and includes a very quick and fair peer-review system.
71. Cooper M, Tsai X, Bennett P. Combination CCNU and vinblastine chemotherapy for canine mast cell tumours: 57 cases. Vet Comp Oncol. 2009;7(3):196-206.

72. Rassnick KM, Bailey DB, Flory AB, et al. Efficacy of vinblastine for treatment of canine mast cell tumors. J Vet Intern Med. 2008;22(6):1390-1396.

73. Rassnick KM, Moore AS, Williams LE, et al. Treatment of canine mast cell tumors with CCNU (lomustine). J Vet Intern Med. 1999;13(6): 601-605.

74. Vail DM, von Euler H, Rusk AW, et al. A randomized trial investigating the efficacy and safety of water soluble micellar paclitaxel (Paccal Vet) for treatment of nonresectable grade 2 or 3 mast cell tumors in dogs. J Vet Intern Med. 2012;26(3):598-607.

75. Veterinary Cooperative Oncology Group. Common terminology criteria for adverse events (VCOG-CTCAE) following chemotherapy or biological antineoplastic therapy in dogs and cats v1.1. Vet Comp Oncol. Epub July 20, 2011.

76. Bailey DB, Rassnick KM, Kristal O, Chretin JD, Balkman CE. Phase I dose escalation of single-agent vinblastine in dogs. J Vet Intern Med. 2008;22(6):1397-1402.

77. Kristal O, Rassnick KM, Gliatto JM, et al. Hepatotoxicity associated with CCNU (lomustine) chemotherapy in dogs. $J$ Vet Intern Med. 2004;18(1):75-80.

78. Skorupski KA, Hammond GM, Irish AM, et al. Prospective randomized clinical trial assessing the efficacy of Denamarin for prevention of CCNU-induced hepatopathy in tumor-bearing dogs. $J$ Vet Intern Med. 2011;25(4):838-845.

79. Hahn KA, Oglivie G, Rusk T, et al. Masitinib is safe and effective for the treatment of canine mast cell tumors. JVet Intern Med. 2008;22(6):1301-1309.

80. Hahn KA, Legendre AM, Shaw NG, et al. Evaluation of 12- and 24-month survival rates after treatment with masitinib in dogs with nonresectable mast cell tumors. Am J Vet Res. 2010;71(11):1354-1361.

81. London CA, Hannah AL, Zadovoskaya R, et al. Phase I dose-escalating study of SU11654, a small molecule receptor tyrosine kinase inhibitor, in dogs with spontaneous malignancies. Clin Cancer Res. 2003;9(7):2755-2768.

82. London CA, Malpas PB, Wood-Follis SL, et al. Multi-center, placebo-controlled, double-blind, randomized study of oral toceranib phosphate (SU11654), a receptor tyrosine kinase inhibitor, for the treatment of dogs with recurrent (either local or distant) mast cell tumor following surgical excision. Clin Cancer Res. 2009;15(11): 3856-3865.

83. Bernabe LF, Portela R, Nguyen S, et al. Evaluation of the adverse event profile and pharmacodynamics of toceranib phosphate administered to dogs with solid tumors at doses below the maximum tolerated dose. BMC Vet Res. 2013;9:190

84. Mitchell L, Thamm DH, Biller BJ. Clinical and immunomodulatory effects of toceranib combined with low-dose cyclophosphamide in dogs with cancer. J Vet Intern Med. 2012;26(2):355-362.

85. Robat $\mathrm{C}$, London $\mathrm{C}$, Bunting L, et al. Safety evaluation of combination vinblastine and toceranib phosphate (Palladia ${ }^{\circledR}$ ) in dogs: a phase I dose-finding study. Vet Comp Oncol. 2012;10(3):174-183.
Visit http://www.dovepress.com/testimonials.php to read real quotes from published authors. 